\title{
STRONG HOMOLOGY IS NOT ADDITIVE
}

\author{
S. MARDEŠIĆ AND A. V. PRASOLOV
}

\begin{abstract}
Using the continuum hypothesis $(\mathrm{CH})$ we show that strong homology groups $\bar{H}_{p}(X)$ do not satisfy Milnor's additivity axiom. Moreover, $\mathrm{CH}$ implies that strong homology does not have compact supports and that $\bar{H}_{p}(X)$ need not vanish for $p<0$.
\end{abstract}

1. Introduction. Generalizing classical Steenrod homology (see [18]) Ju. T. Lisica and S. Mardešić [5-10] have defined strong homology groups $\bar{H}_{p}(X, A ; G)$, $p \geq 0$, for arbitrary pairs of spaces $(X, A)$. These groups have many desirable properties. In particular, they satisfy all the Eilenberg-Steenrod axioms on pairs $(X, A)$, where $X$ is paracompact and $A$ is closed [10]. They are invariants of strong shape $[\mathbf{1 0}]$ and vanish if $p$ exceeds the shape dimension sd $X[\mathbf{1 5}]$. Moreover, under very general assumptions, strong homology groups satisfy the relative homeomorphism axiom and the wedge axiom [20] and, therefore, for metric compacta coincide with Steenrod homology groups. For spaces having the homotopy type of CW complexes strong homology groups coincide with singular groups [10].

Following J. Milnor [18], we say that a homology theory $H_{*}$ is additive provided for every family of topological spaces $\left(X^{\alpha}, \alpha \in A\right)$ the natural inclusions $i^{\alpha}: X^{\alpha} \rightarrow$ $\amalg_{\alpha \in A} X^{\alpha}$ of $X^{\alpha}$ into the topological sum $\amalg X^{\alpha}$ induce an isomorphism of groups

$$
\psi: \bigoplus_{\alpha \in A} H_{p}\left(X^{\alpha}\right) \rightarrow H_{p}\left(\coprod_{\alpha \in A} X^{\alpha}\right), \quad p \in \mathbf{Z} .
$$

If $H_{*}$ is a homology theory and $X$ is an arbitrary space one can consider the direct system $\left(H_{p}(K), i_{K K^{\prime *}}\right)$, where $K$ ranges over all compact subsets of $X$ and $i_{K K^{\prime}}: K \rightarrow K^{\prime}$ are the inclusion maps, $K \subseteq K^{\prime}$. We say that $H_{*}$ has compact supports if the inclusions $K \rightarrow X$ induce an isomorphism

$$
H_{p}^{c}(X)=\operatorname{colim}\left(H_{p}(K), i_{K K^{\prime *}}\right) \rightarrow H_{p}(X), \quad p \in \mathbf{Z} .
$$

In this paper we consider the following questions.

QUESTION 1. Is strong homology additive?

QUESTION 2. Does strong homology (of locally compact finite-dimensional spaces) have compact supports?

Received by the editors April 20, 1987.

1980 Mathematics Subject Classification (1985 Revision). Primary 55N07, 55N40, 55P55, 55U99, $18 \mathrm{E} 25$.

Key words and phrases. Strong homology, Steenrod homology, additivity, derived functors, inverse limits, derived limits, strong shape.

This paper was written during the winter semester of 1986/87 while A. V. Prasolov, on leave from the V. I. Lenin Byelorussian State University in Minsk, USSR, was visiting the University of Zagreb. 
We construct simple examples for which we show, using the continuum hypothesis $(\mathrm{CH})$, that both questions have negative answers.

Since we will be using Z. R. Miminoshvili's version of strong homology [19] (which agrees with [5-10] for $p>0$ but allows groups $\bar{H}_{p}$ with negative $p$ ), the following question naturally arises also.

Question 3. Is $\bar{H}_{p}(X ; G)=0$ for $p<0$ ?

This question too is answered in the negative, using $\mathrm{CH}$.

We gratefully acknowledge help received from Petr Simon of Charles University in Prague, who showed us how to settle a set-theoretic question to which we reduced our problems (see Theorem 2).

2. The examples. Main results. Let $k \geq 0$ be an integer and let $Y^{(k)}$ be a countably infinite compact bouquet of copies of the $k$-sphere $S^{k}$ ( $k$-dimensional Hawaiian earring),

$$
Y^{(k)}=\bigvee_{j=0}^{\infty} S^{k} .
$$

Let $X^{(k)}$ be the topological sum of a countable infinite collection of copies of $Y^{(k)}$,

$$
X^{(k)}=\coprod_{i=0}^{\infty} Y^{(k)}
$$

Note that $X^{(k)}$ is a $k$-dimensional locally compact separable metric space.

In $\S 6$ we will compute the strong homology groups $\bar{H}_{p}$ of $Y^{(k)}$ and $X^{(k)}$ (integer coefficients $\mathbb{Z}$ ) for all $p$ and $k$. In this computation a certain pro-Abelian group $\mathbf{A}$ plays an essential role. It is defined as follows. Let $\mathbb{N}=\{0,1, \ldots\}$ be the set of all nonnegative integers and let $\mathbb{N}^{\mathbb{N}}$ be the set of all sequences $n=(n(0), n(1), \ldots, n(i), \ldots), n(i) \in \mathbb{N}$. We order $\mathbb{N}^{\mathbb{N}}$ coordinatewise, i.e., we put $n \leq m$ provided $n(i) \leq m(i)$ for every $i \in \mathbb{N}$. Clearly, $\mathbb{N}^{\mathbb{N}}$ is a directed ordered set. For every $n \in \mathbb{N}^{\mathbb{N}}$ we put

$$
A_{n}=\bigoplus_{i=0}^{\infty} \bigoplus_{j=0}^{n(i)} \mathbb{Z}
$$

and we take for $p_{n m}: A_{m} \rightarrow A_{n}, n \leq m$, the natural projection.

Computation of strong homology groups (integer coefficients), performed in $\S 6$, includes the following results.

PROPOSITION 1. For $p>0$,

$$
\bar{H}_{p}\left(Y^{(p+1)}\right)=0 .
$$

PROPOSITION 2. For $p>0$,

$$
\bar{H}_{p}\left(X^{(p+1)}\right)=\lim ^{1} \mathbf{A}
$$

where $\lim ^{1} \mathbf{A}$ denotes the first derived limit of $\mathbf{A}$.

PROPOSITION 3.

$$
\bar{H}_{-1}\left(X^{(0)}\right)=\lim ^{1} \mathbf{A} .
$$


Proposition 1 shows that

$$
\bigoplus_{i=0}^{\infty} \bar{H}_{p}\left(Y^{(p+1)}\right)=0
$$

Moreover, since finite additivity is an easy consequence of the Eilenberg-Steenrod axioms, we also conclude that

$$
\bar{H}_{p}\left(\coprod_{i=0}^{k} Y^{(p+1)}\right)=0, \quad k \in \mathbb{N}, p>0 .
$$

Therefore, for strong homology with compact supports we have

$$
\bar{H}_{p}^{c}\left(X^{(p+1)}\right)=0 .
$$

We see, by (4), (7), (9) and (6), that there exist examples answering Questions 1-3 in the negative, provided one can answer affirmatively the next question.

QUESTION 4. Is $\lim ^{1} \mathbf{A} \neq 0$ ?

We will now state an equivalent set-theoretic question.

Let $U, V$ be arbitrary subsets of $N \times N$ and let $f: U \rightarrow \mathbb{Z}, g: V \rightarrow \mathbb{Z}$ be arbitrary functions. We say that $f$ and $g$ almost coincide, and we write $f \equiv g$, whenever the set

$$
\{(i, j) \in U \cap V: f(i, j) \neq g(i, j)\}
$$

is finite.

QUESTION 5. Let $\left(f_{n}, n \in \mathbb{N}^{\mathbb{N}}\right)$ be a collection of functions $f_{n}: U_{n} \rightarrow \mathbb{Z}$, where

$$
U_{n}=\{(i, j) \in \mathbb{N} \times \mathbb{N}: 0 \leq j \leq n(i)\} .
$$

If $f_{n} \equiv f_{m}$ for any pair $n, m \in \mathbb{N}^{\mathbb{N}}$, does there exist a function $f: \mathbb{N} \times \mathbb{N} \rightarrow \mathbb{Z}$ such that $f \equiv f_{n}$ for every $n \in \mathbb{N}^{\mathbb{N}}$ ?

In $\S 8$ we will prove the following theorem.

THEOREM 1. $\lim ^{1} \mathbf{A}=0$ if and only if Question 5 has an affirmative answer.

In $\S 9$ we will give a proof (following P. Simon) of the following result.

THEOREM 2. The continuum hypothesis $(\mathrm{CH})$ implies a negative answer to Question 5. Therefore, $(C H)$ implies $\lim ^{1} \mathbf{A} \neq 0$.

Hence, Propositions 1-3 and Theorems 1 and 2 establish our main result.

THEOREM 3. Assuming the continuum hypothesis, Questions 1, 2 and 3 have negative answers.

REMARK (added in the revised version). After this paper was submitted for publication A. Dow, P. Simon and J. Vaughan showed that the proper forcing axiom implies a positive answer to Question 5 , and thus implies $\lim ^{1} \mathbf{A}=0$. This means that the question whether the strong homology group $\bar{H}_{p}\left(X^{p+1}\right)$ of the space $X^{p+1}, p>0$, vanishes or not is undecidable in set theory based on the ZFC-axioms. A paper of these authors entitled Strong homology and the proper forcing axiom is in preparation (verbal communication from J. Vaughan). 
3. Strong homology of inverse systems. In order to define strong homology of spaces, we need strong homology $\bar{H}^{p}(\mathbf{C})$ of inverse systems of chain complexes $\mathbf{C}=\left(C_{\lambda}, p_{\lambda \lambda^{\prime}}, \Lambda\right)$ over preordered sets $(\Lambda, \leq)$. We will also need higher derived limits $\lim ^{n} \mathbf{C}$ of pro-Abelian groups $\mathbf{C}$. Both concepts can be defined using the notion of a cosimplicial replacement $R^{\cdot} \mathbf{C}[\mathbf{1}, \mathbf{2}]$, where $\mathbf{C}$ is an inverse system in the category $C=C h$ of chain complexes or the category $C=A b$ of Abelian groups. $R^{\cdot} \mathrm{C}$ is a cosimplicial chain complex (Abelian group) defined by

$$
R^{m} \mathbf{C}=\prod_{\lambda_{0} \leq \cdots \leq \lambda_{m}} C_{\lambda_{0}}, \quad m=0,1, \ldots
$$

To define the coface operators $\delta^{p}: R^{m-1} \mathbf{C} \rightarrow R^{m} \mathbf{C}, i=0,1, \ldots, m$, it suffices to define the compositions $\pi_{\lambda} \delta^{p}$, where $\lambda=\left(\lambda_{0} \leq \cdots \leq \lambda_{m}\right)$ and $\pi_{\lambda}$ is the natural projection of $R^{m} \mathbf{C}$ to the corresponding factor. If $i>0$, we put

$$
\pi_{\lambda} \delta^{i}=\pi_{\lambda_{i}}
$$

where $\lambda_{i}$ is obtained from $\lambda$ by deleting $\lambda_{i}$. If $i=0$, we put

$$
\pi_{\lambda} \delta^{0}=p_{\lambda_{0} \lambda_{1}} \pi_{\lambda_{0}} \text {. }
$$

The codegeneracy operators $\sigma^{i}: R^{m+1} \mathbf{C} \rightarrow R^{m} \mathbf{C}, i=0,1, \ldots, m$ (which we will not need), are defined by defining $\pi_{\lambda} \sigma^{i}$. For every $i$ we put $\pi_{\lambda} \sigma^{i}=\pi_{\lambda^{i}}$, where $\lambda^{i}$ is obtained from $\lambda$ by repeating $\lambda_{i}$. The usual conditions on coface and codegeneracy operators are readily verified.

In the case of pro-Abelian groups, we can make $R^{\cdot} \mathbf{C}$ into a cochain complex by defining the coboundary operator $\delta: R^{m-1} \mathbf{C} \rightarrow R^{m} \mathbf{C}$ by

$$
\delta=\sum_{i=0}^{m}(-1)^{i} \delta^{i}
$$

It is known $[\mathbf{1}, \mathbf{3}]$ that the cohomology of this cochain complex yields the derived limits of $\mathbf{C}$,

$$
H^{m}\left(R^{\cdot} \mathbf{C}\right) \approx \lim ^{m} \mathbf{C} .
$$

In the case of inverse systems of chain complexes we can make $R^{\cdot} \mathrm{C}$ into a bicomplex by putting

$$
R^{p q}(\mathbf{C})=R^{p}\left(\mathbf{C}_{-q}\right)=\prod_{\lambda_{0} \leq \cdots \leq \lambda_{p}}\left(C_{\lambda_{0}}\right)_{-q}, \quad p \geq 0 .
$$

For $p<0$ we put $R^{p q}(\mathbf{C})=0$.

Beside the differential $\delta$ from (4) we also have the differential $\partial$ from the chain complexes $C_{\lambda}$.

With the bicomplex $\left(R^{p q}(\mathbf{C}), \delta, \partial\right)$ we associate the cochain complex $K(\mathbf{C})$ defined by

$$
K_{n}(\mathbf{C})=\prod_{p+q=n} R^{p q}(\mathbf{C}), \quad n \in \mathbb{Z}
$$

the differential $d: K_{n}(\mathbf{C}) \rightarrow K_{n+1}(\mathbf{C})$ is given by

$$
(-1)^{p}(d x)_{\lambda}=\partial\left(x_{\lambda}\right)-(\delta x)_{\lambda}, \quad \lambda=\left(\lambda_{0}, \ldots, \lambda_{p}\right), \lambda_{0} \leq \cdots \leq \lambda_{p} .
$$


Note that the total complex $K=K(\mathbf{C})$ is defined using direct products and not direct sums (which is more often the case).

By definition, strong homology of $\mathbf{C}$ is the cohomology of $(K, d)=(K(\mathbf{C}), d)$,

$$
\bar{H}_{n}(\mathbf{C})=H^{-n}(K), \quad n \in \mathbb{Z} .
$$

If $\mathbf{X}=\left(X_{\lambda}, p_{\lambda \lambda^{\prime}}, \Lambda\right)$ is an inverse system of spaces and $G$ is an Abelian group, we associate with it the inverse system of singular chain complexes

$$
S \mathbf{X}=\left(S\left(X_{\lambda}\right), p_{\lambda \lambda^{\prime} \#}, \Lambda\right)
$$

and the inverse system

$$
S \mathbf{X} \otimes G=\left(S\left(X_{\lambda}\right) \otimes G, p_{\lambda \lambda^{\prime} \#} \otimes 1, \Lambda\right) .
$$

Then the strong homology group $\bar{H}_{n}(\mathbf{X} ; G)$ with coefficients in $G$ is defined as the strong group of the inverse system of chain complexes $S \mathbf{X} \otimes G$.

REMARK 1. This definition coincides with the definition from [7] if $n>0$. For $n<0$ one had in $[\mathbf{7}] C_{n}=C_{n}(\mathbf{X} ; G)=0$ and therefore $\bar{H}_{n}(X, G)=0$. If one wants to obtain for $n=0$ the same groups as in [19], one must modify the definitions in [7] by introducing a nontrivial group $C_{-1}=C_{-1}(\mathbf{X} ; G)$ as the image of the boundary operator $d: K_{0} \rightarrow K_{1}$.

REMARK 2. This section as well as the next one can be easily generalized by replacing inverse systems $\mathrm{C}$ by functors $F: I \rightarrow C$ from a small category $I$ to the category $\mathrm{Ch}$ or $\mathrm{Ab}$.

4. The Miminoshvili exact sequences. The computation of strong homology groups is usually not an easy task. In some cases it can be performed by computing $s$-stage strong homology groups $\bar{H}_{n}^{(s)}(\mathbf{C})$, introduced by Ju. T. Lisica [4] and Z. R. Miminoshvili [19]. To define these groups one considers the quotient complexes $K^{(s)}(\mathbf{C})$ of $K=K(\mathbf{C}), s \geq 0$, where

$$
\left.K_{n}^{(s)}(\mathbf{C})=\prod_{\substack{p+q=n \\ p \leq s}} R^{p q}(\mathbf{C})=\prod_{p \leq s \lambda_{0} \leq \cdots \leq \lambda_{p}} \prod_{\lambda_{\lambda_{0}}}\right)_{p-n} .
$$

Then

$$
\begin{gathered}
H_{n}^{(s)}(\mathbf{C})=H^{-n}\left(K^{(s)}(\mathbf{C})\right), \\
\bar{H}_{n}^{(s)}(\mathbf{C})=\operatorname{Im}\left(j_{n}^{s, s+1}\right),
\end{gathered}
$$

where the homomorphisms $j_{n}^{s, s+1}: H_{n}^{(s+1)}(\mathbf{C}) \rightarrow H_{n}^{(s)}(\mathbf{C})$ are induced by the natural projections $K^{(s+1)}(\mathbf{C}) \rightarrow K^{(s)}(\mathbf{C})$. Clearly, these homomorphisms induce homomorphisms $\bar{j}_{n}^{s-1, s}: \bar{H}_{n}^{(s)}(\mathbf{C}) \rightarrow \bar{H}_{n}^{(s-1)}(\mathbf{C})$.

REMARK 3. For $s=0$ the group $\bar{H}_{n}^{(0)}(\mathbf{C})$ is isomorphic to $\lim H_{n}(\mathbf{C})$, where

$$
H_{n}(\mathbf{C})=\left(H_{n}\left(C_{\lambda}\right), p_{\lambda \mu *}, \Lambda\right) .
$$

The desired isomorphism $\bar{H}_{n}^{(0)}(\mathbf{C}) \rightarrow \lim H_{n}(\mathbf{C})$ is obtained as follows. An arbitrary element $u$ of $\bar{H}_{n}^{(0)}(\mathbf{C})$ is the $j_{n}^{01}$-image of an element of $H_{n}^{(1)}(\mathbf{C})$, which is given by a cocycle $x$ of $K_{-n}^{(1)}(\mathbf{C})$. This cocycle consists of chains $x_{\lambda_{0}} \in\left(C_{\lambda_{0}}\right)_{n}$ and 
$x_{\lambda_{0} \lambda_{1}} \in\left(C_{\lambda_{0}}\right)_{n+1}, \lambda_{0} \leq \lambda_{1}$, such that $\partial x_{\lambda_{0}}=0, \partial x_{\lambda_{0} \lambda_{1}}=p_{\lambda_{0} \lambda_{1}} x_{\lambda_{1}}-x_{\lambda_{0}}$. Therefore, the homology class $\left[x_{\lambda_{0}}\right] \in H_{n}\left(C_{\lambda_{0}}\right)$ is defined and $p_{\lambda_{0} \lambda_{1 *}}\left[x_{\lambda_{1}}\right]=\left[x_{\lambda_{0}}\right]$, which shows that $\left(\left[x_{\lambda_{0}}\right]\right), \lambda_{0} \in \Lambda$, is an element of $\lim H_{n}(\mathbf{C})$. We assign this element to $u$ (for more details see $[\mathbf{1 5}]$ ).

The $s$-stage strong groups $\bar{H}_{n}^{(s)}$ and the strong groups $\bar{H}_{n}$ are connected by exact sequences, announced by Z. R. Miminoshvili [19]. We state these sequences in the following two theorems.

THEOREM 4. For every integer $n$ there exists an exact sequence

$$
\begin{aligned}
& 0 \rightarrow \lim ^{1} H_{n+1}(\mathbf{C}) \rightarrow \bar{H}_{n}^{(1)}(\mathbf{C}) \rightarrow \bar{H}_{n}^{(0)}(\mathbf{C}) \rightarrow \lim ^{2} H_{n+1}(\mathbf{C}) \rightarrow \cdots \\
& \quad \ldots \rightarrow \lim ^{s} H_{n+1}(\mathbf{C}) \rightarrow \bar{H}_{n-s+1}^{(s)}(\mathbf{C}) \rightarrow \bar{H}_{n-s+1}^{(s-1)}(\mathbf{C}) \rightarrow \lim ^{s+1} H_{n+1}(\mathbf{C}) \rightarrow \cdots
\end{aligned}
$$

THEOREM 5. For every integer $n$ there exists an exact sequence

$$
0 \rightarrow \lim ^{1} \bar{H}_{n+1}^{(s)}(\mathbf{C}) \rightarrow \bar{H}_{n}(\mathbf{C}) \rightarrow \lim \bar{H}^{(s)}(\mathbf{C}) \rightarrow 0 .
$$

In (6) $\lim$ and $\lim ^{1}$ are applied to the towers $\left(\bar{H}_{m}^{(0)}(\mathbf{C}) \leftarrow H_{m}^{(1)}(\mathbf{C}) \leftarrow \cdots\right)$, where $m=n$ and $n+1$ respectively.

The referee has informed the authors that a different proof of Theorems 4 and 5 as well as of Corollaries 1 and 2 will appear in [17].

For any inverse system of chain complexes $\mathbf{C}$ and $s \geq 0$ we define subcomplexes

$$
\Gamma^{(s)}(\mathbf{C})=\operatorname{Ker}\left(K^{(s)}(\mathbf{C}) \rightarrow K^{(s-1)}(\mathbf{C})\right)
$$

and groups

$$
\begin{aligned}
& D^{s t}(\mathbf{C})=H^{s+t}\left(K^{(s)}(\mathbf{C})\right), \\
& E^{s t}(\mathbf{C})=H^{s+t}\left(\Gamma^{(s)}(\mathbf{C})\right) .
\end{aligned}
$$

Clearly,

$$
\Gamma_{n}^{(s)}(\mathbf{C})=\prod_{\lambda_{0} \leq \cdots \leq \lambda_{s}}\left(C_{\lambda_{0}}\right)_{s-n}
$$

and the differential of the complex $\Gamma^{(s)}(\mathbf{C})$ is given by $\Pi(-1)^{s} \partial$. Therefore,

$$
E^{s t}=\prod_{\lambda_{0} \leq \cdots \leq \lambda_{s}} H_{-t}\left(C_{\lambda_{0}}\right) .
$$

Consider the short exact sequence of cochain complexes

$$
0 \rightarrow \Gamma^{(s)}(\mathbf{C}) \rightarrow K^{(s)}(\mathbf{C}) \rightarrow K^{(s-1)}(\mathbf{C}) \rightarrow 0
$$

with obvious morphisms. The corresponding long exact sequence of cohomology groups can be interpreted as an exact couple of bigraded Abelian groups.
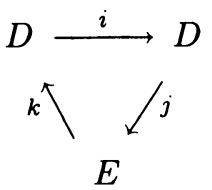
where $D^{s t}$ and $E^{\text {st }}$ are given by (8) and (9) respectively and $i, j$ and $k$ have bidegrees $(-1,1),(1,0)$ and $(0,0)$.

We now consider the derived couple
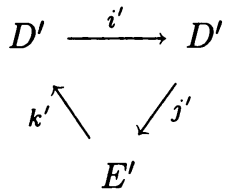

of the exact couple (13) (see [11]). Then $D^{\prime}=i D, E^{\prime}=\operatorname{Ker}(j k) / \operatorname{Im}(j k), i^{\prime}=i \mid D^{\prime}$, $k^{\prime}$ is induced by $k$ and $j^{\prime}$ is induced by $j i^{-1}$. The bidegree of $i^{\prime}$ is $(-1,1)$, of $k^{\prime}$ is $(0,0)$ and of $j^{\prime}$ is $(2,-1)$. Moreover,

$$
\begin{gathered}
D^{\prime s t}=\operatorname{Im}\left(D^{s+1, t-1} \rightarrow D^{s, t}\right) \\
=\operatorname{Im}\left(H_{-s-t}^{(s+1)}(\mathbf{C}) \rightarrow H_{-s-t}^{(s)}(\mathbf{C})\right)=\bar{H}_{-s-t}^{(s)}(\mathbf{C}), \\
E^{\prime s t}=\lim ^{s} H_{-t}(\mathbf{C}) .
\end{gathered}
$$

In order to obtain (16) first note that

$$
{ }^{\prime} E^{s t}=\operatorname{ker}\left(E^{s t} \stackrel{j k}{\rightarrow} E^{s+1, t}\right) / \operatorname{Im}\left(E^{s-1, t} \stackrel{j k}{\rightarrow} E^{s t}\right) .
$$

If we show that $j k: E^{s t} \rightarrow E^{s+1, t}$ coincides (up to sign) with $\delta$ (see $\S 3(4)$ ),

$$
\delta: \prod_{\lambda_{0} \leq \cdots \leq \lambda_{s}} H_{-t}\left(C_{\lambda_{0}}\right) \rightarrow \prod_{\lambda_{0} \leq \cdots \leq \lambda_{s+1}} H_{-t}\left(C_{\lambda_{0}}\right),
$$

(16) will follow from the definition of $\lim ^{s}$.

(11) shows that the domain and codomain of the two maps coincide. An element of $E^{s t}$ is a cohomology class $[x] \in H^{s+t}\left(\Gamma^{(s)}(\mathbf{C})\right)$, where $x \in \Gamma^{(s)}(\mathbf{C}) \subseteq K^{(s)}(\mathbf{C})$ is given by $x_{\lambda_{0} \cdots \lambda_{i}}, 0 \leq i \leq s$, and $x_{\lambda_{0}}=\cdots=x_{\lambda_{0} \cdots \lambda_{s-1}}=0, d x=0$. Consequently, $(\delta x)_{\lambda_{0} \cdots \lambda_{s}}=0$, so that $d x=0$ implies $\partial\left(x_{\lambda_{0} \cdots \lambda_{s}}\right)=0$. Now $k[x]=[x] \in H^{s+t}\left(K^{(s)}(\mathbf{C})\right)$. Since $j$ is the boundary homomorphism of the homology sequence, $j k[x]=[d y] \in H^{s+t+1}\left(K^{(s+1)}(\mathbf{C})\right)$, where $y \in K^{(s+1)}(\mathbf{C})$ and $y_{\lambda_{0} \cdots \lambda_{i}}=x_{\lambda_{0} \cdots \lambda_{i}}, 0 \leq i \leq s$. Clearly, $(d y)_{\lambda_{0} \cdots \lambda_{i}}=0$ for $0 \leq i \leq s$. Moreover, $(\delta y)_{\lambda_{0} \cdots \lambda_{s+1}}=(\delta x)_{\lambda_{0} \cdots \lambda_{s+1}}$. Therefore,

$$
(-1)^{s}[d y]_{\lambda_{0} \cdots \lambda_{s+1}}=\left[(\delta x)_{\lambda_{0} \cdots \lambda_{s+1}}\right]
$$

as desired.

We obtain from (14) the long exact sequence

$$
\begin{aligned}
& 0=D^{\prime-1,-n} \rightarrow E^{1,-n-1} \rightarrow D^{1,-n-1} \rightarrow D^{\prime 0,-n} \rightarrow \cdots \\
& \quad \cdots \rightarrow E^{\prime s,-n-1} \rightarrow D^{\prime s,-n-1} \rightarrow D^{\prime s-1,-n} \rightarrow E^{\prime s+1,-n-1} \rightarrow \cdots .
\end{aligned}
$$

Using (15) and (16) we see that (17) coincides with (5).

In order to prove Theorem 5, we use this well-known fact (see, e.g. [16, Theorem A.19]). 
LEMMA 1. Let $\mathbf{C}=\left(C_{m}, p_{m m+1}\right)$ be a tower of epimorphisms between cochain complexes and let $C=\lim \mathbf{C}$. Then there is an exact sequence

$$
0 \rightarrow \lim ^{1} H^{n-1}(\mathbf{C}) \rightarrow H^{n}(C) \rightarrow \lim H^{n}(\mathbf{C}) \rightarrow 0, \quad n \in \mathbb{Z} .
$$

Note that the short exact sequence of cochain complexes

$$
0 \rightarrow C \rightarrow \prod_{s \geq 0} C_{s} \stackrel{1-p}{\rightarrow} \prod_{s \geq 0} C_{s} \rightarrow 0
$$

induces a long cohomology sequence

$$
\begin{aligned}
\cdots \rightarrow \prod_{s \geq 0} H^{n-1}\left(C_{s}\right) & \stackrel{1-p}{\rightarrow} \prod_{s \geq 0} H^{n-1}\left(C_{s}\right) \rightarrow H^{n}(C) \\
& \rightarrow \prod_{s \geq 0} H^{n}\left(C_{s}\right) \stackrel{1-p}{\rightarrow} \prod_{s \geq 0} H^{n}\left(C_{s}\right) \rightarrow \cdots .
\end{aligned}
$$

The sequence (18) is readily obtained from (20), because lim is the kernel of $1-p$ and $\lim ^{1}$ is the cokernel of $1-p$.

Application of Lemma 1 to the tower

$$
K^{(0)}(\mathbf{C}) \leftarrow \cdots \leftarrow K^{(s)}(\mathbf{C}) \stackrel{j}{\leftarrow} K^{(s+1)}(\mathbf{C}) \leftarrow \cdots
$$

yields the exact sequence

$$
0 \rightarrow \lim ^{1} H_{n+1}^{(s)}(\mathbf{C}) \rightarrow \bar{H}_{n}(\mathbf{C}) \rightarrow \lim H_{n}^{(s)}(\mathbf{C}) \rightarrow 0, \quad n \in \mathbb{Z} .
$$

It remains to show that (21) implies (6). However, this is an immediate consequence of the definition of $\bar{H}_{n}^{(s)}$ and the following lemma [1, Chapter IX, Proposition $2.2]$.

LEMMA 2. Let $\mathbf{G}=\left(G_{0} \stackrel{p_{1}}{\leftarrow} G_{1} \stackrel{p_{2}}{\leftarrow} \cdots\right)$ be a tower of Abelian groups and let $p \mathbf{G}=\left(p_{1}\left(G_{1}\right) \stackrel{p_{1}}{\leftarrow} p_{2}\left(G_{2}\right) \leftarrow \cdots\right)$. Then the homomorphisms $p_{m}$ induce isomorphisms

$$
\lim \mathbf{G} \approx \lim p \mathbf{G}, \quad \lim ^{1} \mathbf{G} \approx \lim ^{1} p \mathbf{G} .
$$

To prove Lemma 2, consider the cochain complexes

$$
\begin{aligned}
& M=\left(0 \rightarrow M^{0}=\prod_{m \geq 0} G_{m} \stackrel{1-p}{\rightarrow} M^{1}=\prod_{m \geq 0} G_{m} \rightarrow 0\right), \\
& N=\left(0 \rightarrow N^{0}=\prod_{m \geq 1} p G_{m} \stackrel{1-p}{\rightarrow} N^{1}=\prod_{m \geq 1} p G_{m} \rightarrow 0\right),
\end{aligned}
$$

where $(1-p)\left(x_{0}, x_{1}, \ldots\right)=\left(x_{0}-p_{1}\left(x_{1}\right), x_{1}-p_{2}\left(x_{2}\right), \ldots\right)$. Clearly,

$$
\begin{array}{rlrl}
H^{0} M & =\lim \mathbf{G}, & H^{1} M & =\lim ^{1} \mathbf{G}, \\
H^{0} N & =\lim p \mathbf{G}, & H^{1} N=\lim ^{1} p \mathbf{G} .
\end{array}
$$

Therefore, it suffices to show that the cochain mapping $M \rightarrow N$ induced by $p_{m}$ is a cochain homotopy equivalence. This is indeed the case because the inclusions $p_{m}\left(G_{m}\right) \rightarrow G_{m-1}$ induce the homotopy inverse cochain mapping $N \rightarrow M$ (with cochain homotopies given by the identity maps $M^{1} \rightarrow M^{0}$ and $N^{1} \rightarrow N^{0}$ ).

Theorems 4 and 5 imply the following corollaries, used in $\S 6$. 
COROLLARY 1. Let $1 \leq s_{0} \leq s_{1}$ be such that for a given integer $p$ one has

$$
\lim ^{t} H_{p+s}(\mathbf{C})=0, \quad s_{0} \leq s \leq s_{1}, t>0 .
$$

Then the homomorphisms $j_{p}^{t-1, t}$ yield isomorphisms

$$
\bar{H}_{p}^{\left(s_{0}-1\right)}(\mathbf{C}) \approx \cdots \approx \bar{H}_{p}^{(s)}(\mathbf{C}) \approx \cdots \approx \bar{H}_{p}^{\left(s_{1}\right)}(\mathbf{C})
$$

for $s_{0} \leq s \leq s_{1}$.

Proof. (27) implies $\lim ^{s} H_{p+s}(\mathbf{C})=0, \lim ^{s+1} H_{p+s}(\mathbf{C})=0$ for $s_{0} \leq s \leq s_{1}$. Therefore, by (5), the homomorphisms $j_{p}^{s-1, s}, s_{0} \leq s \leq s_{1}$, yield the isomorphisms (28).

COROLLARY 2. Let $s_{0} \geq 1$ be such that for a given integer $p$ one has

$$
\lim ^{t} H_{p+s}(\mathbf{C})=0, \quad s_{0} \leq s, t>0 .
$$

Then the homomorphisms $j_{p}^{s-1, s}$ and $j_{p}^{s}$ induce isomorphisms

$$
\bar{H}_{p}^{\left(s_{0}-1\right)}(\mathbf{C}) \approx \cdots \approx \bar{H}_{p}^{(s)}(\mathbf{C}) \approx \cdots \approx \bar{H}_{p}(\mathbf{C}), \quad s_{0} \leq s .
$$

ProOF. By Corollary 1, in the tower $\left(\bar{H}_{p}^{(0)}(\mathbf{C}) \leftarrow \bar{H}_{p}^{(1)}(\mathbf{C}) \leftarrow \cdots\right)$ the projections $\bar{j}_{p}^{s-1, s}$ are isomorphisms for $s_{0}-1 \leq s$. Therefore,

$$
\lim \bar{H}_{p}^{(s)}(\mathbf{C}) \approx \bar{H}_{p}^{(s)}(C), \quad s_{0}-1 \leq 1 .
$$

Similarly, since also $\lim ^{s} H_{p+s+1}(\mathbf{C})=\lim ^{s+1} H_{p+s+1}(\mathbf{C})=0, s_{0} \leq s$, in the tower $\left(\bar{H}_{p+1}^{(0)}(\mathbf{C}) \leftarrow \bar{H}_{p+1}^{(1)}(\mathbf{C}) \leftarrow \cdots\right)$ the projections $\bar{j}_{p+1}^{s-1, s}$ are isomorphisms for $s \geq s_{0}$. Therefore, the tower is Mittag-Leffler and

$$
\lim ^{1} \bar{H}_{p+1}^{(s)}(\mathbf{C})=0
$$

(see [3] or [14]).

Now (6) implies

$$
\lim \bar{H}_{p}^{(s)}(\mathbf{C}) \approx \bar{H}_{p}(\mathbf{C})
$$

(33) and (31) yield (30).

5. Strong homology of spaces. ANR-resolutions. Following $[5,10]$ we define strong homology of spaces using ANR-resolutions [12]. An ANR-resolution of a space $X$ consists of an inverse system $\mathbf{X}=\left(X_{\lambda}, p_{\lambda \mu}, \Lambda\right)$ of ANRs (for metric spaces) and a system $\mathbf{p}=\left(p_{\lambda}\right)$ of maps $p_{\lambda}: X \rightarrow X_{\lambda}, \lambda \in \Lambda$, such that $p_{\lambda \mu} p_{\mu}=p_{\lambda}$, for $\lambda \leq \mu$. Moreover, $\mathbf{p}$ must satisfy certain approximate factorization conditions (R1), (R2) (see [12] or [14]). Instead of stating these conditions we state here two equivalent conditions (see $[12,14])$ :

(B1) For every normal covering $U$ of $X$ there is a $\lambda \in \Lambda$ and a covering $\mathcal{V}_{\lambda}$ of $X_{\lambda}$ such that $p_{\lambda}^{-1}\left(\mathcal{V}_{\lambda}\right)$ refines $U$.

(B2) For every $\lambda \in \Lambda$ and open set $V \subseteq X_{\lambda}$, which contains $\overline{p_{\lambda}(X)}$, there is a $\mu \geq \lambda$ such that

$$
p_{\lambda \mu}\left(X_{\mu}\right) \subseteq V
$$


It was proved in [6 and 8] that for any two cofinite ANR-resolutions $\mathbf{p}: X \rightarrow \mathbf{X}$, $\mathbf{p}^{\prime}: X \rightarrow \mathbf{X}^{\prime}$ of a space $X$ there is a natural isomorphism $\bar{H}_{p}(\mathbf{X} ; G) \rightarrow \bar{H}_{p}\left(\mathbf{X}^{\prime} ; G\right)$. Therefore, the strong homology group $\bar{H}_{p}(X ; G)$ of the space $X$ was defined as $\bar{H}_{p}(\mathbf{X} ; G)$, where $\mathbf{p}: X \rightarrow \mathbf{X}$ was any cofinite ANR-resolution. It was shown in [13] that in this definition one can also use ANR-resolutions which are not cofinite.

We will now consider ANR-resolutions of topological sums of spaces. Let $\left(X^{\alpha}, \alpha \in A\right)$ be a collection of spaces and let $X=\amalg_{\alpha \in A} X^{\alpha}$. For each $\alpha \in A$ let

$$
\mathbf{p}^{\alpha}=\left(p_{\lambda}^{\alpha}\right): X^{\alpha} \rightarrow \mathbf{X}^{\alpha}=\left(X_{\lambda}^{\alpha}, p_{\lambda \mu}^{\alpha}, \Lambda^{\alpha}\right)
$$

be an ANR-resolution. Let $\Lambda=\prod_{\alpha \in A} \Lambda^{\alpha}$ be ordered by the product ordering $\leq$. That is, if $\lambda=(\lambda(\alpha)), \mu=(\mu(\alpha)) \in \Lambda$, we put $\lambda \leq \mu$ if and only if $\lambda(\alpha) \leq \mu(\alpha)$ for every $\alpha \in A$. For $\lambda=(\lambda(\alpha)) \in \Lambda$ let $X_{\lambda}=\amalg_{\alpha \in A} X_{\lambda(\alpha)}^{\alpha}$. Furthermore, let $p_{\lambda \mu}: X_{\mu} \rightarrow X_{\lambda}, \lambda \leq \mu$, and $p_{\lambda}: X \rightarrow X_{\lambda}, \lambda, \mu \in \Lambda$, be given by

$$
p_{\lambda \mu}\left|X_{\mu(\alpha)}^{\alpha}=p_{\lambda(\alpha) \mu(\alpha)}^{\alpha}: X_{\mu(\alpha)}^{\alpha} \rightarrow X_{\lambda(\alpha)}^{\alpha}, \quad p_{\lambda}\right| X^{\alpha}=p_{\lambda(\alpha)}^{\alpha}: X^{\alpha} \rightarrow X_{\lambda(\alpha)}^{\alpha} .
$$

Clearly, $\mathbf{X}=\left(X_{\lambda}, p_{\lambda \mu}, \Lambda\right)$ is an inverse system of ANRs and $\mathbf{p}=\left(p_{\lambda}\right): X \rightarrow \mathbf{X}$ satisfies the condition $p_{\lambda \mu} p_{\mu}=p_{\lambda}$, for $\lambda \leq \mu$.

THEOREM 6. If each $\mathbf{p}^{\alpha}: X^{\alpha} \rightarrow \mathbf{X}^{\alpha}$ is an ANR-resolution, then $\mathbf{p}: X \rightarrow \mathbf{X}$ is also an ANR-resolution.

PROOF. We must verify conditions (B1) and (B2).

(B1) Let $U$ be a normal covering of $X=\amalg_{\alpha \in A} X^{\alpha}$. Since $\mathcal{U}^{\alpha}=U \mid X^{\alpha}$ is a normal covering of $X^{\alpha}$, there is a $\lambda(\alpha) \in \Lambda^{\alpha}$ and a covering $\mathcal{V}_{\lambda(\alpha)}^{\alpha}$ of $X_{\lambda(\alpha)}^{\alpha}$ such that $\left(p_{\lambda(\alpha)}^{\alpha}\right)^{-1}\left(\mathcal{V}_{\lambda(\alpha)}^{\alpha}\right)$ refines $\mathcal{U}^{\alpha}$. We now put $\lambda=(\lambda(\alpha)) \in \Lambda$ and $\mathcal{V}_{\lambda}=\bigcup_{\alpha} \mathcal{V}_{\lambda(\alpha)}^{\alpha}$. Clearly, $\mathcal{V}_{\lambda}$ is a covering of $X_{\lambda}=\amalg X_{\lambda(\alpha)}^{\alpha}$ and $p_{\lambda}^{-1}\left(\mathcal{V}_{\lambda}\right)$ refines $U$.

(B2) Let $\lambda=(\lambda(\alpha)) \in \Lambda$ and let $V \subseteq X_{\lambda}$ be an open set which contains

$$
\overline{p_{\lambda}(X)}=\bigcup \overline{p_{\lambda(\alpha)}^{\alpha}\left(X^{\alpha}\right)}
$$

Then, for every $\alpha \in A, V^{\alpha}=V \cap X_{\lambda(\alpha)}^{\alpha}$ contains $\overline{p_{\lambda(\alpha)}^{\alpha}\left(X^{\alpha}\right)}$ and, therefore, there is a $\mu(\alpha) \geq \lambda(\alpha)$ such that

$$
p_{\lambda(\alpha) \mu(\alpha)}^{\alpha}\left(X_{\mu(\alpha)}^{\alpha}\right) \subseteq V^{\alpha}, \quad \alpha \in A .
$$

Consequently, $\mu=(\mu(\alpha)) \in \Lambda, \boldsymbol{\mu} \geq \lambda$ and

$$
p_{\lambda \mu}\left(X_{\mu}\right) \subseteq V
$$

REMARK 4. If $A$ and $\Lambda^{\alpha}$ are infinite, $\Lambda$ is not cofinite. In particular, this is the case when $A=N$ and $\Lambda^{\alpha}=N$ for each $\alpha \in N$.

6. Strong homology of the spaces $Y^{(k)}$ and $X^{(k)}$. The aim of this section is to determine the strong homology groups (integer coefficients) of the spaces $Y^{(k)}$ and $X^{(k)}$ defined in $\S 2$.

THEOREM 7. If $k>0$, then

$$
\bar{H}_{p}\left(Y^{(k)}\right) \approx \begin{cases}0, & p \neq 0, k \\ \prod_{j \in \mathbb{N}} \mathbb{Z}, & p=k \\ \mathbb{Z}, & p=0\end{cases}
$$




$$
\bar{H}_{p}\left(Y^{(0)}\right) \approx \begin{cases}0, & p \neq 0 \\ \prod_{j \in \mathbb{N}} \mathbb{Z}, & p=0\end{cases}
$$

THEOREM 8. If $k>0$, then

$$
\bar{H}_{p}\left(X^{(k)}\right) \approx \begin{cases}0, & p>k, \\ \lim \mathbf{A}, & p=k, \\ \lim ^{k-p} \mathbf{A}, & p<k, p \neq 0, \\ \lim ^{k} \mathbf{A} \oplus\left(\bigoplus_{i \in N} \mathbb{Z}\right), & p=0\end{cases}
$$

$$
\bar{H}_{p}\left(X^{(0)}\right) \approx \begin{cases}0, & p>0 \\ \lim \mathbf{A}, & p=0 \\ \lim ^{-p} \mathbf{A}, & p<0 .\end{cases}
$$

PROOF OF THEOREM 7. Let $Y_{n}^{(k)}$ be the wedge of $n+1$ copies of the $k$-spheres

$$
Y_{n}^{(k)}=\bigvee_{j=0}^{n} S^{k}, \quad n \geq 0
$$

Let $p_{m n}: Y_{n}^{(k)} \rightarrow Y_{m}^{(k)}, m \leq n$, and $p_{m}: Y^{(k)} \rightarrow Y_{m}^{(k)}$ be the natural projections. Then $\mathbf{Y}^{(k)}=\left(Y_{n}^{(k)}, p_{m n}\right)$ is an inverse sequence of compact $k$-dimensional ANRs and $p_{m n} p_{n}=p_{m}, m \leq n$. Clearly, $\mathbf{p}=\left(p_{m}\right): Y^{(k)} \rightarrow \mathbf{Y}^{(k)}$ is an inverse limit and since we are dealing with compact spaces, $p$ is an ANR-resolution [14]. Therefore, $\bar{H}_{p}\left(Y^{(k)}\right)$ (integer coefficients) equals $\bar{H}_{p}\left(\mathbf{Y}^{(k)}\right)$ and the Čech homology group

$$
\check{H}_{p}\left(Y^{(k)}\right)=\lim H_{p}\left(\mathbf{Y}^{(k)}\right)=\bar{H}_{p}^{(0)}\left(\mathbf{Y}^{(k)}\right)
$$

(see Remark 3).

Notice that for $k>0$

$$
H_{p}\left(Y_{n}^{(k)}\right)= \begin{cases}0, & p \neq 0, k \\ \bigoplus_{j=0}^{n} \mathbb{Z}, & p=k, \\ \mathbb{Z}, & p=0 .\end{cases}
$$

For $k=0$ we have

$$
H_{p}\left(Y_{n}^{(0)}\right)= \begin{cases}0, & p \neq 0 \\ \mathbb{Z} \oplus \bigoplus_{j=0}^{n} \mathbb{Z}, & p=0 .\end{cases}
$$

Clearly, $p_{m n *}: H_{p}\left(Y_{n}^{(k)}\right) \rightarrow H_{p}\left(Y_{m}^{(k)}\right)$ is the natural projection. Therefore, we find for the Cech homology groups, for $k>0$,

$$
\begin{array}{r}
\check{H}_{p}\left(Y^{(k)}\right) \approx \begin{cases}0, & p \neq 0, k, \\
\prod_{j \in \mathbb{N}} \mathbb{Z}, & p=k, \\
\mathbb{Z}, & p=0 .\end{cases} \\
\check{H}_{p}\left(Y^{(0)}\right) \approx \begin{cases}0, & p \neq 0, \\
\mathbb{Z} \oplus \prod_{j \in \mathbb{N}} \mathbb{Z}, & p=0 .\end{cases}
\end{array}
$$


Since $\left(H_{p}\left(Y_{0}^{(k)}\right) \leftarrow H_{p}\left(Y_{1}^{(k)}\right) \leftarrow \cdots\right)$ is a tower with epimorphic projections, we conclude that condition (29) of $\S 4$ is satisfied for all $p$ and $s \geq 0$. Therefore, by Corollary 2 to Theorem 5 ,

$$
\bar{H}_{p}\left(Y^{(k)}\right) \approx \check{H}_{p}\left(Y^{(k)}\right),
$$

and the groups $\bar{H}_{p}\left(Y^{(k)}\right)$ are given by (8) and (9) as claimed.

REMARK 5. Strong homology groups of $Y^{(k)}$ can also be determined using [18].

PROOF OF THEOREM 8. Let us denote the copies of $Y^{(k)}$ in $X^{(k)}$ by $Y^{i}$ (omitting $k$ ), $i \in \mathbb{N}$, and the copies of $S^{k}$ in $Y^{i}$ by $S_{j}^{i}$. Using for $Y^{i}$ the same resolutions

$$
\mathbf{p}_{m}^{i}=\left(p_{m}^{i}\right): Y^{i} \rightarrow \mathbf{Y}^{i}=\left(Y_{n}^{i}, p_{m n}^{i}\right)
$$

as in the proof of Theorem 7, and applying Theorem 6, we obtain an ANR-resolution $p=\left(p_{a}\right): X^{(k)} \rightarrow \mathbf{X}^{(k)}=\left(X_{a}^{(k)}, p_{a b}, \Lambda\right)$ for $X^{(k)}$. Here $\Lambda=\mathbb{N}^{\mathbb{N}}$ is the set of all functions $a: \mathbb{N} \rightarrow \mathbb{N}$, where $a \leq b$ if and only if $a(i) \leq b(i)$ for all $i \in \mathbb{N}$.

$$
X_{a}^{(k)}=\coprod_{i \in \mathbb{N}} Y_{a(i)}^{i},
$$

$p_{a b}: X_{b}^{(k)} \rightarrow X_{a}^{(k)}, a \leq b$, is $\amalg_{i \in \mathbb{N}} p_{a(i) b(i)}^{i}$ and $p_{a}: X^{(k)} \rightarrow X_{a}^{(k)}$ is $\amalg_{i \in \mathbb{N}} p_{a(i)}^{i}$.

By (6) and (7), for $k>0$,

$$
H_{p}\left(X_{a}^{(k)}\right)= \begin{cases}0, & p \neq 0, k, \\ \bigoplus_{i \in \mathbb{N}} \bigoplus_{j=0}^{a(i)} \mathbb{Z}, & p=k, \\ \bigoplus_{i \in \mathbb{N}} \mathbb{Z}, & p=0,\end{cases}
$$

and for $k=0$,

$$
H_{p}\left(X_{a}^{(0)}\right)= \begin{cases}0, & p \neq 0 \\ \left(\bigoplus_{i \in \mathbb{N}} \mathbb{Z}\right) \oplus\left(\bigoplus_{i \in \mathbb{N}} \bigoplus_{j=0}^{a(i)} \mathbb{Z}\right), & p=0 .\end{cases}
$$

This menas that for $k>0$ the pro-group $H_{p}\left(\mathbf{X}^{(k)}\right)$ is given by

$$
H_{p}\left(\mathbf{X}^{(k)}\right)= \begin{cases}0, & p \neq 0, k \\ \mathbf{A}, & p=k \\ \bigoplus_{i \in \mathbb{N}} \mathbb{Z}, & p=0\end{cases}
$$

and for $k=0$ by

$$
H_{p}\left(\mathbf{X}^{(0)}\right)= \begin{cases}0, & p \neq 0, \\ \bigoplus_{i \in \mathbf{N}} \mathbb{Z} \oplus \mathbf{A}, & p=0,\end{cases}
$$

where $\mathbf{A}$ is the pro-group described in $\S 2$.

We will first prove (3) for $k>0$. If $p \geq k$, then, by $(14), H_{p+s}\left(\mathbf{X}^{(k)}\right)=0$ for $s \geq 1$ and therefore, by Corollary $2, \bar{H}_{p}\left(\mathbf{X}^{(k)}\right)=\bar{H}_{p}^{(0)}\left(\mathbf{X}^{(k)}\right)=\lim H_{p}\left(\mathbf{X}^{(k)}\right)$. Consequently, (14) implies

$$
\bar{H}_{p}\left(\mathbf{X}^{(k)}\right)= \begin{cases}0, & p>k \\ \lim \mathbf{A}, & p=k\end{cases}
$$


Now assume that $p<k$. Then $H_{p+s}\left(\mathbf{X}^{(k)}\right)=0$ for $s \geq k-p+1>1$. Therefore, by Corollary 2,

$$
\bar{H}_{p}\left(\mathbf{X}^{(k)}\right) \approx \bar{H}_{p}^{(k-p)}\left(\mathbf{X}^{(k)}\right), \quad p<k .
$$

Moreover, by (14), $H_{n}\left(\mathbf{X}^{(k)}\right)=0$, for $n<k, n \neq 0$, and $H_{0}\left(\mathbf{X}^{(k)}\right)$ is the constant pro-group $\bigoplus_{i \in \mathbb{N}} \mathbb{Z}$. Therefore,

$$
\lim ^{t} H_{p+s}\left(\mathbf{X}^{(k)}\right)=0, \quad \text { for } s \leq k-p-1, t>0 .
$$

By Corollary 1 , for $p+1<k$, we have

$$
\bar{H}_{p}^{(k-p-1)}\left(\mathbf{X}^{(k)}\right) \approx \bar{H}_{p}^{(0)}\left(\mathbf{X}^{(k)}\right)= \begin{cases}0, & p \neq 0 \\ \bigoplus_{i \in \mathbf{N}} \mathbb{Z}, & p=0 .\end{cases}
$$

For $p+1=k,(19)$ is obvious.

Now consider the exact sequence $\S 4(5)$ of Theorem 4 , for $n=k-1$. Putting $s=k-p$ and using (17) and (19), one concludes that

$$
\bar{H}_{p}\left(\mathbf{X}^{(k)}\right)=\lim ^{k-p} \mathbf{A}, \quad p<k, p \neq 0,-1 .
$$

For $p=0,-1, \S 4(5)$ yields the exact sequence

$$
0 \rightarrow \lim ^{k} \mathbf{A} \rightarrow \bar{H}_{0}\left(\mathbf{X}^{(k)}\right) \stackrel{\varphi}{\rightarrow} \bigoplus_{i \in N} \mathbb{Z} \rightarrow \lim ^{k+1} \mathbf{A} \rightarrow \bar{H}_{-1}\left(\mathbf{X}^{(k)}\right) \rightarrow 0 .
$$

However, $\varphi$ is an epimorphism because the homomorphism $\psi$ from $\S 1(1)$ is a right inverse of $\varphi$, i.e. $\varphi \psi$ is the identity on $\bigoplus_{i \in N} \mathbb{Z}$. Therefore, (21) yields a split exact sequence

$$
0 \rightarrow \lim ^{k} \mathbf{A} \rightarrow \bar{H}_{0}\left(\mathbf{X}^{(k)}\right) \stackrel{\varphi}{\rightarrow} \bigoplus_{i \in N} \mathbb{Z} \rightarrow 0
$$

and the exact sequence

$$
0 \rightarrow \lim ^{k+1} \mathbf{A} \rightarrow \bar{H}_{-1}\left(\mathbf{X}^{(k)}\right) \rightarrow 0 \text {. }
$$

(22) and (23) imply (3) in the cases $p=0,-1$.

Now consider the case $k=0$. Clearly, for every $p$ and $s>-p$ or $s<-p$ and for $t>0$ we have

$$
\lim ^{t} H_{p+s}\left(\mathbf{X}^{(0)}\right)=0
$$

because $H_{p+s}\left(\mathbf{X}^{(0)}\right)=0$. Applying again Corollaries 1 and 2 we obtain

$$
\begin{gathered}
\bar{H}_{p}\left(\mathbf{X}^{(0)}\right) \approx H_{p}^{(0)}\left(\mathbf{X}^{(0)}\right)=0, \quad p>0 ; \\
\bar{H}_{p}\left(\mathbf{X}^{(0)}\right) \approx H_{p}^{(-p)}\left(\mathbf{X}^{(0)}\right), \quad p \leq 0 ; \\
\bar{H}_{p}^{(-p-1)}\left(\mathbf{X}^{(0)}\right)=H_{p}^{(0)}\left(\mathbf{X}^{(0)}\right)=0, \quad p<0 .
\end{gathered}
$$

The exact sequence $\S 4(5)$ for $n=-1$ now yields

$$
\bar{H}_{p}\left(\mathbf{X}^{(0)}\right)=\lim ^{-p}(\mathbf{A}) \text {. }
$$




\section{Additivity of Cech homology groups.}

\section{THEOREM 9. Čech homology groups are additive.}

This is asserted in [16]. However, in the literature we could not find a proof, so we give one here.

Let $\left(X^{\alpha}, \alpha \in A\right)$ be a collection of spaces and let $X=\amalg_{\alpha \in A} X^{\alpha}$ be their topological sum. We must show that the inclusions $i^{\alpha}: X^{\alpha} \rightarrow X$ induce isomorphisms

$$
i_{*}: \bigoplus \check{H}_{p}\left(X^{\alpha}\right) \rightarrow \check{H}_{p}\left(\coprod_{\alpha} X^{\alpha}\right),
$$

determined by $i_{*}^{\alpha}: \check{H}_{p}\left(X^{\alpha}\right) \rightarrow \check{H}_{p}(X)$.

\section{LEMMA 3. $i_{*}$ is a monomorphism.}

PROOF. We first consider the case of two summands $X=X^{1} \amalg X^{2}$. We choose points $a^{1} \in X^{1}, a^{2} \in X^{2}$. Let $r^{1}: X \rightarrow X^{1}$ be defined by

$$
\begin{aligned}
& r^{1} \mid X^{1}=\mathrm{id}, \\
& r^{1} \mid X^{2}=a^{1} .
\end{aligned}
$$

Analogously we define $r^{2}: X \rightarrow X^{2}$. Note that $r^{1} i^{1}=$ id and therefore $r_{*}^{1} i_{*}^{1}=$ id, which shows that $i_{*}^{1}$ is a monomorphism. Similarly, $i_{*}^{2}$ is a monomorphism.

If $u=\left(u^{1}, u^{2}\right) \in \check{H}_{p}\left(X^{1}\right) \oplus \check{H}_{p}\left(X^{2}\right)$ is such that $i_{*}(u)=0$, i.e., $i_{*}^{1}\left(u^{1}\right)+i_{*}^{2}\left(u^{2}\right)=$ 0 , then $r_{*}^{1} i_{*}^{1}\left(u^{1}\right)+r_{*}^{1} i_{*}^{2}\left(u^{2}\right)=0$. If $p \neq 0, r_{*}^{1} i_{*}^{2}\left(u^{2}\right)=0=\check{H}_{p}\left(\left\{a^{1}\right\}\right)$ and we see that $u^{1}=0$. Analogously, $u^{2}=0$ so that $u=0$.

If $p=0, r_{*}^{1} i_{*}^{2}\left(u^{2}\right)$ is of the form $-j_{*}^{1} g_{*}^{1}\left[a^{1}\right]$, where $j^{1}:\left\{a^{1}\right\} \rightarrow X^{1}$ is the inclusion, $g^{1} \in G$ and $\left[a^{1}\right]$ is the class of $\breve{H}_{0}\left(\left\{a^{1}\right\}\right)$, determined by the point $a^{1}$. In this case $u^{1}=j_{*}^{1} g^{1}\left[a^{1}\right]$. Similarly, $u^{2}=j_{*}^{2} g^{2}\left[a^{2}\right]$. Since $\left\{a^{1}, a^{2}\right\}$ is a retract of $X=X^{1} \amalg X^{2}$, the inclusion $j:\left\{a^{1}, a^{2}\right\} \rightarrow X$ induces a monomorphism $\check{H}_{0}\left(\left\{a^{1}, a^{2}\right\}\right) \rightarrow \check{H}_{0}(X)$. Note that

$$
\begin{aligned}
0 & =i_{*}^{1}\left(u^{1}\right)+i_{*}^{2}\left(u^{2}\right)=i_{*}^{1} j_{*}^{1} g^{1}\left[a^{1}\right]+i_{*}^{2} j_{*}^{2} g^{2}\left[a^{2}\right] \\
& =j_{*}\left(k_{*}^{1} g^{1}\left[a^{1}\right]+k_{*}^{2} g^{2}\left[a^{2}\right]\right),
\end{aligned}
$$

where $k^{1}:\left\{a^{1}\right\} \rightarrow\left\{a^{1}, a^{2}\right\}, k^{2}:\left\{a^{2}\right\} \rightarrow\left\{a^{1}, a^{2}\right\}$ are inclusions. Consequently,

$$
k^{1} g^{1}\left[a^{1}\right]+k^{2} g^{2}\left[a^{2}\right]=0 .
$$

Now, it suffices to conclude that $g^{1}\left[a^{1}\right]=g^{2}\left[a^{2}\right]=0$, because this will imply $u^{1}=u^{2}=0$. However, this assertion follows from the fact that Lemma 3 holds for the sum $\left\{a^{1}, a^{2}\right\}=\left\{a^{1}\right\} \amalg\left\{a^{2}\right\}$.

The proof for finitely many summands is obtained by induction. In the general case we first need to observe that every element $u$ of $\bigoplus_{\alpha \in A} \check{H}_{p}\left(X^{\alpha}\right)$ is contained in a finite sum $\bigoplus_{\alpha=1}^{n} \breve{H}_{p}\left(X^{\alpha}\right)$ and that $i_{*}$ restricted to this sum factors through $\check{H}_{p}\left(\amalg_{\alpha=1}^{n} X^{\alpha}\right)$. However, the inclusion $\amalg_{\alpha=1}^{n} X^{\alpha} \rightarrow \amalg_{\alpha \in A} X^{\alpha}$ induces a monomorphism on $\check{H}_{p}$.

REMARK 6. Lemma 3 also applies to strong homology because its proof uses only functoriality of the homology groups and the fact that for polyhedra (onepoint and two-point sets) these groups agree with singular homology groups and therefore are additive. 
PROOF OF THEOREM 9. It remains to prove that $i_{*}$ is an epimorphism. Let $p^{\alpha}=\left(p_{\lambda}^{\alpha}\right): X^{\alpha} \rightarrow \mathrm{X}^{\alpha}$ be an ANR-resolution of $X^{\alpha}, \alpha \in A$, and let $p=\left(p_{\lambda}\right): X \rightarrow$ $\mathbf{X}$ be the ANR-resolution defined in $\S 5$ (see Theorem 6). Since

$$
\check{H}_{p}\left(X^{\alpha}\right)=\lim H_{p}\left(\mathbf{X}^{\alpha}\right) \quad \text { and } \quad \check{H}_{p}(X)=\lim H_{p}(\mathbf{X})
$$

we must prove that the homomorphism

$$
i_{*}: \bigoplus_{\alpha \in A} \lim H_{p}\left(\mathbf{X}^{\alpha}\right) \rightarrow \lim H_{p}(\mathbf{X})
$$

is an epimorphism.

An arbitrary element $v$ of $\lim H_{p}(\mathbf{X})$ is given by a collection $\left(v_{\lambda}\right), \lambda \in \Lambda, \Lambda=$ $\prod_{\alpha \in A} \Lambda^{\alpha}$, where $v_{\lambda} \in H_{p}\left(X_{\lambda}\right)$ and

$$
p_{\lambda \mu}\left(v_{\mu}\right)=v_{\lambda}, \quad \lambda \leq \mu .
$$

We must find a finite set $\left\{\alpha_{1}, \ldots, \alpha_{n}\right\} \subseteq A$ and elements $v^{\alpha_{j}} \in \lim H_{p}\left(\mathbf{X}^{\alpha_{j}}\right)$, $j=1, \ldots, n$, such that

$$
i^{\alpha_{1}}\left(v^{\alpha_{1}}\right)+\cdots+i^{\alpha_{n}}\left(v^{\alpha_{n}}\right)=v .
$$

Let $x_{\lambda}$ be a singular $p$-cycle of $X_{\lambda}=\amalg X_{\lambda(\alpha)}^{\alpha}$, which belongs to the class $v_{\lambda}$. There are uniquely determined $p$-cycles $x_{\lambda}^{\alpha}$ of $X_{\lambda(\alpha)}^{\alpha}$ such that

$$
x_{\lambda}=\sum_{\alpha \in A} x_{\lambda}^{\alpha} .
$$

Moreover, for a given $\lambda \in \Lambda$ there are only finitely many $\alpha \in A$ for which $x_{\lambda}^{\alpha} \neq 0$. Put

$$
B=\left\{\alpha \in A: \exists \lambda \in \Lambda, 0 \neq\left[x_{\lambda}^{\alpha}\right] \in H_{p}\left(X_{\lambda(\alpha)}^{\alpha}\right)\right\} .
$$

We will show that $B$ is a finite set.

We first prove that whenever for some $\lambda, \lambda^{\prime} \in \Lambda$ and for a given $\alpha \in A$ we have $\lambda(\alpha)=\lambda^{\prime}(\alpha)$, then

$$
\left[x_{\lambda}^{\alpha}\right]=\left[x_{\lambda^{\prime}}^{\alpha}\right] .
$$

Indeed, one can choose $\mu \in \Lambda, \mu \geq \lambda, \lambda^{\prime}$. Then, by (6),

$$
\begin{aligned}
p_{\lambda \mu}\left[x_{\mu}\right] & =\left[x_{\lambda}\right], \\
p_{\lambda^{\prime} \mu} & {\left[x_{\mu}\right]=\left[x_{\lambda^{\prime}}\right], }
\end{aligned}
$$

which implies

$$
\begin{gathered}
p_{\lambda(\alpha) \mu(\alpha)}^{\alpha}\left[x_{\mu}^{\alpha}\right]=\left[x_{\lambda}^{\alpha}\right], \\
p_{\lambda^{\prime}(\alpha) \mu(\alpha)}^{\alpha}\left[x_{\mu}^{\alpha}\right]=\left[x_{\lambda^{\prime}}^{\alpha}\right] .
\end{gathered}
$$

Since $\lambda(\alpha)=\lambda^{\prime}(\alpha)$, the left-hand sides of (13) and (14) coincide.

Now choose for every $\alpha \in B$ some $\lambda_{\alpha} \in \Lambda$ such that $\left[x_{\lambda_{\alpha}}^{\alpha}\right] \neq 0$. Define $\lambda \in \Lambda$ so that

$$
\lambda(\alpha)=\lambda_{\alpha}(\alpha), \quad \alpha \in B
$$

for $\alpha \in A \backslash B, \lambda(\alpha) \in \Lambda^{\alpha}$ is arbitrary. Applying (10) to $\lambda$ and $\lambda_{\alpha}$ we see that

$$
\left[x_{\lambda}^{\alpha}\right]=\left[x_{\lambda_{\alpha}}^{\alpha}\right] \neq 0, \quad \alpha \in B
$$

because $\lambda(\alpha)=\lambda_{\alpha}(\alpha)$. 
Now (16) implies $x_{\lambda}^{\alpha} \neq 0$ for $\alpha \in B$. However, we already observed that for an arbitrary $\lambda \in \Lambda$ the set of all $\alpha \in A$, for which $x_{\lambda}^{\alpha} \neq 0$, is finite. Therefore, $B$ must be finite.

Let $B=\left\{\alpha_{1}, \ldots, \alpha_{n}\right\}$. For any $j \in\{1, \ldots, n\}$ we will now define $v^{\alpha_{j}} \in$ $\lim H_{p}\left(\mathbf{X}^{\alpha_{j}}\right)$ so that $(7)$ holds. For any $\lambda_{j} \in \Lambda^{\alpha_{j}}$ we define $\left(v^{\alpha_{j}}\right)_{\lambda_{j}}$ as $\left[x_{\lambda}^{\alpha_{j}}\right] \in$ $H_{p}\left(X_{\lambda_{j}}^{\alpha_{j}}\right)$, where $\lambda \in \Lambda$ is such that $\lambda\left(\alpha_{j}\right)=\lambda_{j}$. By $(10),\left(v^{\alpha_{j}}\right)_{\lambda_{j}}$ does not depend on the particular choice of $\lambda$ but only on $\alpha_{j}$ and $\lambda_{j}$. If $\lambda_{j} \leq \lambda_{j}^{\prime}$, one can assume that $\lambda \leq \lambda^{\prime}$. Then, by (12), $\left[x_{\lambda}\right]=p_{\lambda \lambda^{\prime}}\left[x_{\lambda^{\prime}}\right]$ and therefore

$$
\left(v^{\alpha_{j}}\right)_{\lambda_{j}}=p_{\lambda_{j} \lambda_{j}^{\prime}}^{\alpha_{j}}\left(v^{\alpha_{j}}\right)_{\lambda_{j}^{\prime}}
$$

This shows that $\left(\left(v^{\alpha_{j}}\right)_{\lambda_{j}}\right) \in \lim H_{p}\left(\mathbf{X}^{\alpha_{j}}\right)$.

Notice that for every $\lambda \in \Lambda$ and $j \in\{1, \ldots, n\}$ we have

$$
\left(i_{*}^{\alpha_{j}}\left(v^{\alpha_{j}}\right)\right)_{\lambda}=\left(v^{\alpha_{j}}\right)_{\lambda\left(\alpha_{j}\right)}=\left(v^{\alpha_{j}}\right)_{\lambda_{j}}=\left[x_{\lambda}^{\alpha_{j}}\right] .
$$

By (18), (8) and the definition of $B$, we see that

$$
\left(\sum_{j=1}^{n} i^{\alpha_{j}}\left(v^{\alpha_{j}}\right)\right)_{\lambda}=\sum_{\alpha \in B}\left[x_{\lambda}^{\alpha}\right]=\sum_{\alpha \in A}\left[x_{\lambda}^{\alpha}\right]=\left[\sum_{\alpha \in A} x_{\lambda}^{\alpha}\right]=\left[x_{\lambda}\right]=v_{\lambda},
$$

so that (7) holds.

REMARK 7. Applying Theorem 10 to $X^{(k)}=\amalg_{i \in \mathbb{N}} Y^{(k)}$, we see (by $\S 6(16)$ ) that $\lim \mathbf{A}=\bigoplus_{i \in \mathbb{N}} \prod_{j \in \mathbb{N}} \mathbb{Z}$.

8. Proof of Theorem 1. Along with the pro-group A (defined in $\S 2$ ) we will consider here also a pro-group $\mathbf{B}$ defined as follows. $\mathbf{B}=\left(B_{n}, q_{m n}, \mathbb{N}^{\mathbb{N}}\right)$, where

$$
B_{n}=\prod_{i \in \mathbb{N}} \prod_{j=0}^{n(i)} \mathbb{Z}
$$

and $q_{m n}: B_{n} \rightarrow B_{m}, m \leq n$, are the natural projections. We will need the following lemma.

LEMMA 4.

$$
\lim ^{p} \mathbf{B}= \begin{cases}0, & p \neq 0 \\ \prod_{(i, j) \in \mathbb{N} \times \mathbb{N}} \mathbb{Z}, & p=0 .\end{cases}
$$

ProOF. By the explicit description of $\lim ^{p}$ (see (3(1))), we know that $\lim ^{p} \mathbf{B}$ is the $p$ th cohomology group of the cochain complex $R^{\cdot} \mathbf{B}$, where

$$
R^{p} \mathbf{B}=\prod_{n_{0} \leq \ldots \leq n_{p}} \prod_{i \in N} \prod_{j=0}^{n_{0}(i)} \mathbb{Z}
$$

Clearly, (3) can also be written as

$$
R^{p} \mathbf{B}=\prod_{i \in \mathbb{N}} \prod_{j \in \mathbb{N}} C^{p}(i, j)
$$

where

$$
C^{p}(i, j)=\prod_{\substack{n_{0} \leq \cdots \leq n_{p} \\ 0 \leq j \leq n_{0}(i)}} \mathbb{Z}
$$


The coboundary operator $\delta$ in $R^{*} \mathbf{B}$ induces a coboundary operator in $C^{*}(i, j)$ making $C^{*}(i, j)$ into a cochain complex such that $R^{*} \mathbf{B}$ is a product of the complexes $C^{*}(i, j),(i, j) \in \mathbb{N} \times \mathbb{N}$. Since $H^{p}$ commutes with products, we see that

$$
\lim ^{p} \mathbf{B}=H^{p}\left(R^{\cdot} \mathbf{B}\right)=\prod_{(i, j) \in \mathbb{N} \times \mathbb{N}} H^{p}\left(C^{\cdot}(i, j)\right)
$$

To determine $H^{p}\left(C^{\cdot}(i, j)\right)$, consider the set $\Gamma(i, j) \subseteq \mathbb{N}^{\mathbb{N}}$ of all $n \in \mathbb{N}^{N}$ such that $0 \leq j \leq n(i)$. Let $\mathbf{Z}(i, j)$ be the constant pro-group $\mathbb{Z}$ indexed by $\Gamma(i, j)$. Then

$$
R^{*}(\mathbf{Z}(i, j))=C^{*}(i, j)
$$

so that

$$
\lim ^{p} \mathbf{Z}(i, j)=H^{p}\left(R^{*}(\mathbf{Z}(i, j))\right)=H^{p}\left(C^{*}(i, j)\right) .
$$

Since $\mathbf{Z}(i, j)$ is a constant inverse system we conclude (by [3, Theorem 1.8]) that

$$
\lim ^{p} \mathbf{Z}(i, j)= \begin{cases}0, & p \neq 0 \\ \mathbb{Z}, & p=0 .\end{cases}
$$

Now, (6), (8) and (9) yield the desired formula (2).

We now prove Theorem 1. First note that $\mathbf{A} \subseteq \mathbf{B}$, i.e., $A_{n} \subseteq B_{n}$ is a subgroup for every $n \in \mathbb{N}^{\mathbb{N}}$ and $q_{m n} \mid A_{n}=p_{m n}, m \leq n$. Let $\mathbf{B} / \mathbf{A}=\left(B_{n} / A_{n}, r_{m n}, \mathbb{N}^{N}\right)$, where $r_{m n}$ is the induced homomorphism. Clearly,

$$
0 \rightarrow \mathbf{A} \rightarrow \mathbf{B} \rightarrow \mathbf{B} / \mathbf{A} \rightarrow 0
$$

is a short exact sequence of inverse systems of Abelian groups. Therefore, we have a long exact sequence

$$
0 \rightarrow \lim \mathbf{A} \rightarrow \lim \mathbf{B} \rightarrow \lim \mathbf{B} / \mathbf{A} \rightarrow \lim ^{1} \mathbf{A} \rightarrow \lim ^{1} \mathbf{B} \rightarrow \cdots
$$

Since $\lim ^{1} \mathbf{B}=0$ (Lemma 4), we see that $\lim ^{1} \mathbf{A}=0$ if and only if $\lim \mathbf{B} \rightarrow \lim \mathbf{B} / \mathbf{A}$ is a surjection.

Also by (2), we see that $\lim \mathbf{B}$ is the set of all functions $f: \mathbb{N} \times \mathbb{N} \rightarrow \mathbb{Z}$. For $n \in \mathbb{N}^{\mathbb{N}}$ let $U_{n}=\{(i, j) \in \mathbb{N} \times \mathbb{N}: 0 \leq j \leq n(i)\}$. Elements of $B_{n} / A_{n}$ are classes of functions $f_{n}: U_{n} \rightarrow \mathbb{Z}$, where $f_{n}, f_{n}^{\prime}: U_{n} \rightarrow \mathbb{Z}$ are in the same class $\left[f_{n}\right]=\left[f_{n}^{\prime}\right]$ whenever $f_{n}-f_{n}^{\prime} \in A_{n}$, i.e., $f_{n}$ and $f_{n}^{\prime}$ almost coincide. Therefore, elements of $\lim \mathbf{B} / \mathbf{A}$ can be interpreted as families $\left(\left[f_{n}\right]\right)$ of classes of functions $f_{n}: U_{n} \rightarrow \mathbb{Z}$, $n \in \mathbb{N}^{N}$, such that $f_{m} \equiv f_{n}$ for $m \leq n$. It is now clear that $\lim \mathbf{B} \rightarrow \lim \mathbf{B} / \mathbf{A}$ is a surjection if and only if the answer to Question 5 is affirmative.

9. Proof of Theorem 2. We first prove a simple lemma.

Lemma 5. Let $n^{k}, k \in \mathbb{N}$, be a sequence of functions $n^{k}: \mathbb{N} \rightarrow \mathbb{N}$. Then there exists a function $n: \mathbb{N} \rightarrow \mathbb{N}$ such that for every $k \in \mathbb{N}$ the set

$$
\left\{i \in \mathbb{N}: n^{k}(i) \geq n(i)\right\}
$$

is finite, i.e., $n_{k}$ is almost $<n$. 
ProOF. Put $n(i)=\max \left\{n^{0}(i), \ldots, n^{i}(i)\right\}+1$. Fix an element $k \in \mathbb{N}$. For $i \geq k$ we have $n(i) \geq 1+n^{k}(i)>n^{k}(i)$. Therefore, the set (1) is contained in $\{0,1, \ldots, k-1\}$.

LEMMA 6. Let $\left(m^{\alpha}\right), 0 \leq \alpha<\omega_{1}$, be an $\omega_{1}$-sequence of elements from $\mathbb{N}^{\mathbb{N}}$. Then there exists an $\omega_{1}$-sequence $\left(n^{\alpha}\right), 0<\alpha<\omega_{1}$, of elements from $\mathbb{N}^{\mathbb{N}}$ such that

(i) each $n^{\alpha}: \mathbb{N} \rightarrow \mathbb{N}$ (strictly) increases;

(ii) for $\beta<\alpha$ the set $\left\{i \in \mathbb{N}: n^{\beta}(i) \geq n^{\alpha}(i)\right\}$ is finite;

(iii) for each $0<\alpha<\omega_{1}$ the set $\left\{i \in \mathbb{N}: m^{\alpha}(i) \geq n^{\alpha}(i)\right\}$ is finite.

PROOF. We define the functions $n^{\alpha}$ by transfinite induction. We take for $n^{0}$ any increasing function with $n^{0}(i)>m^{0}(i)$ for every $i \in \mathbb{N}$.

Let $\alpha<\omega_{1}$ and assume that we have already defined $n^{\beta}$ for $\beta<\alpha$ so that (i)-(iii) are fulfilled. We define $n^{\alpha}$ as follows. First note that $\left\{n^{\beta}: 0 \leq \beta<\alpha\right\}$ is a countable collection of functions $\mathbb{N} \rightarrow \mathbb{N}$. By Lemma 5 , there is a function $n: \mathbb{N} \rightarrow \mathbb{N}$ such that the sets $\left\{i \in \mathbb{N}: m^{\beta}(i) \geq n(i)\right\}$ and $\left\{i \in \mathbb{N}: n^{\beta}(i) \geq n(i)\right\}$ are finite for every $0 \leq \beta<\alpha$. We choose for $n^{\alpha}$ any increasing function $n^{\alpha} \geq \max \left(n, m^{\alpha}\right)$.

LEMMA 7. Let $\left(n^{\alpha}: 0 \leq \alpha<\omega_{1}\right)$ be elements of $\mathbb{N}^{\mathbb{N}}$ satisfying (i) and (ii) of Lemma 6 and let $g_{\alpha}: \mathbb{N} \times \mathbb{N} \rightarrow \mathbb{Z}, 0 \leq \alpha<\omega_{1}$, be a collection of functions. Then there exist functions $f_{\alpha}: U_{n^{\alpha}} \rightarrow \mathbb{Z}, 0 \leq \alpha<\omega_{1}$, such that $f_{\beta} \equiv f_{\alpha}$ for any $\beta<\alpha$ and $f_{\alpha} \not \equiv g_{\alpha}$ for each $0 \leq \alpha<\omega_{1}$.

PROOF. We define the functions $f_{\alpha}$ by transfinite induction. We choose for $f_{0}$ any function $U_{n^{0}} \rightarrow \mathbb{Z}$ which differs from $g_{0}$ at infinitely many points $(i, j) \in U_{0}$. Assume that we have already defined $f_{\beta}$ for $0 \leq \beta<\alpha$ in agreement with the requirements. In defining $f_{\alpha}$ we distinguish two cases.

Case 1. $\alpha=\beta+1$. By (ii) in Lemma 6, the set $\left\{i \in \mathbb{N}: n^{\beta}(i)<n^{\alpha}(i)\right\}$ is infinite. Therefore, $U_{n^{\alpha}} \backslash U_{n^{\beta}}$ is infinite. Put

$$
f_{\alpha}(i, j)= \begin{cases}f_{\beta}(i, j), & (i, j) \in U_{n^{\beta}}, \\ g_{\alpha}(i, j)+1, & (i, j) \in U_{n^{\alpha}} \backslash U_{n^{\beta}} .\end{cases}
$$

Clearly, $f_{\alpha} \not \equiv g_{\alpha}$ and $f_{\alpha} \equiv f_{\beta^{\prime}}$ for all $\beta^{\prime} \leq \beta$.

Case 2. $\alpha$ has no immediate predecessor. Then one can find a sequence $\beta_{1}<$ $\beta_{2}<\cdots<\alpha$ with $\lim \beta_{n}=\alpha$. We define (by induction on $i$ ) an increasing sequence of integers $k_{1}<\cdots<k_{i}<\cdots$ with the property that the following sets are contained in the segment $\left[0, k_{i}\right]$ :

$$
\begin{aligned}
& \left\{k \in \mathbb{N}: n^{\beta_{j}}(k) \geq n^{\beta_{l}}(k)\right\}, \quad j<l \leq i, \\
& \left\{k \in \mathbb{N}: n^{\beta_{i}}(k) \geq n^{\alpha}(k)\right\}, \\
& \left\{k \in \mathbb{N}: \exists j, l \leq i, \exists(k, m) \in U_{n^{\beta_{j}}} \cap U_{n^{\beta_{l}}}, f_{\beta_{j}}(k, m) \neq f_{\beta_{l}}(k, m)\right\} .
\end{aligned}
$$

The construction of this sequence is possible because, by properties (i) and (ii) from Lemma 6 and by the induction hypothesis, (3)-(5) is a finite collection of finite subsets of $\mathbb{N}$.

We now define $f_{\alpha}(k, l)$, for $k_{i}<k \leq k_{i+1}$, by

$$
f_{\alpha}(k, l)= \begin{cases}f_{\beta^{i-1}}(k, l), & \text { for } 0 \leq l \leq n^{\beta_{i-1}}(k), \\ f_{\beta^{i}}(k, l), & \text { for } n^{\beta_{i-1}}(k)<l \leq n^{\beta_{i}}(k), \\ g_{\alpha}(k, l)+1, & \text { for } n^{\beta_{i}}(k)<l \leq n^{\alpha}(k) .\end{cases}
$$


For each $i \in\{0,1,2, \ldots\}$ the set

$$
\left\{(k, l) \in U_{n^{\beta_{i}}} \cap U_{n^{\alpha}}: f_{\alpha}(k, l) \neq f_{\beta_{i}}(k, l)\right\}
$$

is contained in the set $\left[0, k_{i}\right] \times\left[0, n^{\alpha}\left(k_{i}\right)\right]$ and is therefore finite. Consequently, $f_{\alpha} \equiv f_{\beta_{i}}$. On the other hand, the set

$$
\left\{(k, l) \in U_{n^{\alpha}}: f_{\alpha}(k, l) \neq g_{\alpha}(k, l)\right\}
$$

contains infinitely many points of the form $\left(k, n^{\beta_{i}}(k)+1\right)$, where $k_{i} \leq k \leq k_{i+1}$ and therefore, $f_{\alpha} \not \equiv g_{\alpha}$ as desired. This concludes the proof of Lemma 7 .

In order to prove Theorem 2 we now assume the continuum hypothesis $\aleph_{1}=2^{\aleph_{0}}$. Therefore, there is a bijection $\alpha \mapsto m^{\alpha}$ between the set of ordinals $\left\{\alpha: 0 \leq \alpha<\omega_{1}\right\}$ and the set $\mathbb{N}^{\mathbb{N}}$. Similarly there is a bijection $\alpha \mapsto g_{\alpha}$ between the same set of ordinals and the set of all functions $\mathbb{N} \times \mathbb{N} \rightarrow \mathbb{Z}$. By Lemma 6 , we choose an $\omega_{1}$-sequence $\left(n^{\alpha}\right), 0 \leq \alpha<\omega_{1}$, satisfying conditions (i)-(iii). Let $\left(f_{\alpha}\right)$ be the $\omega_{1}$ sequence of functions $f_{\alpha}: U_{n^{\alpha}} \rightarrow \mathbb{Z}$ from Lemma 7. Finally, for each $0 \leq \alpha<\omega_{1}$ we define a function $h_{\alpha}: U_{m^{\alpha}} \rightarrow \mathbb{Z}$ by

$$
h_{\alpha}(k, l)= \begin{cases}f_{\alpha}(k, l), & (k, l) \in U_{n^{\alpha}} \cap U_{m^{\alpha}}, \\ 0, & \text { otherwise. }\end{cases}
$$

It is now clear that $h_{\alpha} \equiv h_{\beta}$ and $h_{\alpha} \not \equiv g_{\alpha}$ for arbitrary $0 \leq \alpha, \beta<\omega_{1}$. This completes the proof of Theorem 2 .

\section{REFERENCES}

1. A. K. Bousfield and D. M. Kan, Homotopy limits, completions and localizations, Lecture Notes in Math., vol. 304, Springer-Verlag, Berlin and New York, 1972.

2. J. M. Cordier, Quelques notes sur les théories d'homologie de Steenrod-Sitnikov, Manuscripta Math. 59 (1987), 35-52.

3. C. U. Jensen, Les foncteurs dérivés de $\lim _{\longleftarrow}$ et leurs applications en théorie des modules, Lecture Notes in Math., vol. 254, Springer-Verlag, Berlin and New York, 1972.

4. Ju. T. Lisica, Duality theorems and dual shape and coshape categories, Dokl. Akad. Nauk SSSR 263 (1982), 532-536. (Russian)

5. Ju. T. Lisica and S. Mardesić, Steenrod-Sitnikov homology for arbitrary spaces, Bull. Amer. Math. Soc. (N.S.) 9 (1983), 207-210.

6. __ Coherent prohomotopy and strong shape theory, Glasnik Mat. 19(39) (1984), 335-399.

7. __ Strong homology of inverse systems of spaces. I, Topology Appl. 19 (1985), $29-43$.

8. __ Strong homology of inverse systems of spaces. II, Topology Appl. 19 (1985), 45-64.

9. __ Strong homology of inverse systems. III, Topology Appl. 20 (1985), 29-37.

10. __ Steenrod homology, Geometric and Algebraic Topology, Banach Center Publ., vol. 18, PWN, Warsaw, 1986, pp. 329-343.

11. S. Mac Lane, Homology, Springer-Verlag, Berlin and New York, 1963.

12. S. Mardesic, Approximate polyhedra, resolutions of maps and shape fibrations, Fund. Math. 114 (1981), 53-78.

13. __ A note on strong homology of inverse systems, Tsukuba J. Math. 11 (1987), 177-197.

14. S. Mardesic and J. Segal, Shape theory, North-Holland, Amsterdam, 1982.

15. S. Mardešic and T. Watanabe, Strong homology and dimension, Topology Appl. 29 (1988).

16. W. S. Massey, Homology and cohomology theory, M. Dekker, New York and Basel, 1978.

17. L. D. Mdzinarishvili, On total homology, Geometric and Algebraic Topology, Banach Center Publ., vol. 18, PWN, Warsaw, 1986, pp. 345-361. 
18. J. Milnor, On axiomatic homology theory, Pacific J. Math. 12 (1962), 337-341.

19. Z. Miminoshvili, On the sequence of exact and half-exact homologies of arbitrary spaces, Soobsc. Akad. Nauk Gruzin. SSR 113, No. 1 (1984), 41-44. (Russian)

20. T. Watanabe, Cech homology, Steenrod homology and strong homology. I, Glasnik Mat. 22(42) (1987), 187-238.

Department of Mathematics, University of Zagreb, 41001 Zagreb, P. O. Box 187, YUGOSLAVIA

FaCUlty of Mechanics and Mathematics, V. I. Lenin Byelorussian State UNIVERSITY, 220080 MINSK, USSR 